\title{
Cultivares de batata para sistemas orgânicos de produção
}

\author{
Fabrício Rossi $^{1,3}$; Paulo César T Melo ${ }^{1}$; Joaquim A Azevedo Filho² ${ }^{2}$ Edmilson J Ambrosano ${ }^{3}$; Nivaldo \\ Guirado $^{3}$; Eliana A Schammass ${ }^{4}$; Laís F Camargo ${ }^{3}$ \\ 'USP-ESALQ, C. Postal 9, 13418-900 Piracicaba-SP; ${ }^{2}$ Pólo APTA Leste Paulista, Monte Alegre do Sul-SP; ${ }^{3 P o ́ l o ~ A P T A ~ C e n t r o-S u l ~ C . ~}$ \\ Postal 28, Piracicaba-SP; ${ }^{4}$ Instituto de Zootecnia, Nova Odessa-SP; rossi@apta.sp.gov.br, rossi@agrohomeopatia.com.br
}

\section{RESUMO}

Informações a respeito de cultivares adaptadas ao sistema de cultivo orgânico são escassas. O objetivo deste estudo foi avaliar, sob sistema de cultivo orgânico, genótipos nacionais e estrangeiros desenvolvidos para o cultivo convencional, quanto ao potencial produtivo, em condições de campo. O experimento foi conduzido em 2008, no Pólo APTA Leste Paulista, em Monte Alegre do Sul-SP. O delineamento experimental foi em blocos ao acaso, com 18 tratamentos e quatro repetições. Cada parcela foi constituída por 80 batatas-semente, dispostas em quatro linhas de $5 \mathrm{~m}$ de comprimento, espaçadas de $80 \mathrm{~cm}$, com $25 \mathrm{~cm}$ entre tubérculos. Os genótipos avaliados foram Agata, Asterix, Caesar, Cupido, Éden, Melody, Novella e Vivaldi, de origem estrangeira; e Apuã, Aracy, Catucha, IAC Aracy Ruiva, Itararé, Monte Alegre 172, IAC 6090, APTA 16.5, APTA 15.20 e APTA 21.54, nacionais. Foram avaliadas as características de produtividade total e comercial de tubérculos, massa média total e comercial de tubérculos, teor de matéria seca e severidade da pinta-preta (Alternaria solani). Os clones APTA 16.5, APTA 21.54 e IAC 6090, e as cultivares Cupido, Apuã, Itararé e Monte Alegre 172 foram os mais produtivos. 'APTA 21.54' superou os demais em relação a produtividade comercial $\left(18,07 \mathrm{t} \mathrm{ha}^{-1}\right)$, sendo que 'APTA 16.5', 'Cupido', 'IAC 6090' e 'Itararé' formaram o segundo grupo. As maiores massas médias de tubérculos foram apresentadas pelas cultivares Itararé e Cupido. O clone IAC 6090 e as cultivares Aracy e Aracy Ruiva foram as que apresentaram maiores teores de matéria seca, com valor médio de 22,91\%. 'APTA 16.5', 'Apuã', 'Aracy', 'Aracy Ruiva', 'Éden', 'Ibituaçú' e 'Monte Alegre 172' apresentaram alto nível de resistência à pinta-preta. As cultivares Itararé, Apuã e Cupido são adaptadas ao cultivo orgânico, e os clones avançados APTA 16.5, APTA 21.54 e IAC 6090 apresentam potencial de cultivo no sistema orgânico.

Palavras-chave: Solanum tuberosum, Alternaria solani, agroecologia, olericultura orgânica, sistema de manejo sustentável.

\section{ABSTRACT}

\section{Potato cultivars for organic production systems}

Information regarding cultivars adapted to the organic system of production is scarce. The aim of this study was to evaluate in an organic production system, Brazilian and foreign potato genotypes adapted for conventional systems, in terms of quality and yield potential. The experiment was carried out in 2008, at the Agroecological Experimental Station of the Polo APTA Leste Paulista, in Monte Alegre do Sul, São Paulo State, Brazil. A randomized block experimental design was adopted with 18 treatments and four replications. Each plot consisted of 80 seeds, arranged in four rows of $5 \mathrm{~m}$ spaced $0.80 \mathrm{~m}$ and with $0.25 \mathrm{~m}$ between tubers. The treatments consisted of the cultivars Agata, Apuã, Aracy, Asterix, Caesar, Catucha, Cupido, Éden, IAC Aracy Ruiva, Itararé, Melody, Monte Alegre 172, Novella, and Vivaldi, in addition to the advanced clones IAC 6090, APTA 16.5, APTA 15.20 and APTA 21.54. The following parameters were evaluated: yield, marketable yield, average total and marketable tuber mass, dry matter content and severity of potato early blight. The advanced clones APTA 16.5, APTA 21.54, IAC 6090, and the cultivars Cupido, Apuã, Itararé and Monte Alegre 172 showed the highest yield values. 'APTA 21.54' presente the highest marketable yield (18.07 t ha' ${ }^{-1}$, whereas 'APTA 16.5', 'Cupido', 'IAC 6090' and 'Itarare' formed the subsequent group. The highest tuber mass values were presented by the cultivars Itarare and Cupido. The clone IAC 6090 and the cultivars Aracy and Aracy Ruiva showed the highest dry matter content, with an average of 22.91\%. 'APTA 16.5', 'Apuã', 'Aracy', 'Aracy Ruiva', 'Éden', 'Ibituaçú' and 'Monte Alegre 172' exhibited high level of field tolerance to early blight. Potato genotypes Itararé, Apuã and Cupido are, thus, adapted to organic farming, and the advanced clones APTA 16.5, APTA 21.54 and IAC 6090 have potential for cultivation in the organic system.

Keywords: Solanum tuberosum, Alternaria solani, agroecology, organic olericulture, sustainable crop systems.

(Recebido para publicação em 26 de maio de 2010; aceito em 30 de maio de 2011) (Received on May 26, 2010; accepted on May 30, 2011)

O interesse dos consumidores pelos produtos orgânicos, produzidos sem agredir o meio ambiente, tem aumentado consideravelmente nos últimos anos (Maggio et al., 2008). Nesse sentido, tem sido consenso entre pesquisadores e produtores a necessidade de se desenvolver sistemas sustentáveis de produção. A prática de uma agricultura que preserve os recursos naturais e otimize a produtividade tem se tornado cada vez mais necessária, destacando-se o sistema orgânico de produção (Gomes et al., 2008).

Entre as hortaliças cultivadas no Brasil, a batata é a mais importante sob o ponto de vista econômico, com uma produção de 3,44 milhões de toneladas, ocupando uma área de 139 mil hectares em 2009 (IBGE, 2010). A batata é um dos produtos alimentares mais difundidos em todo o mundo, sendo o quarto alimento em importância social e econômica, ficando atrás apenas do arroz, do trigo e do milho. É um dos alimentos capazes de nutrir a crescente população mundial, não apenas como alimento energético, mas também como fonte de proteínas, vitaminas e minerais (Fao, 2008).

A produção de batata orgânica é uma excelente oportunidade de negócio, haja vista a grande demanda pelo produto e a baixa produção, e um grande desafio para os produtores, que ainda dispõem de poucas informações relativas a cul- 
tivares adaptadas ao sistema de cultivo orgânico, bem como às técnicas de manejo cultural e de controle fitossanitário.

Pesquisas relacionadas ao manejo nutricional orgânico da batateira vêm sendo publicadas com certa frequência: Van Delden (2001) estudou o fornecimento de nitrogênio no crescimento e na produtividade da batateira em sistema orgânico, em vista da dificuldade de sincronizar a sua disponibilidade com a demanda durante o crescimento. Silva et al. (2007) obtiveram maiores produtividades de tubérculos quando combinaram a incorporação de crotalária-júncea com a de esterco antes do plantio da batata. Nelson et al. (2009), estudaram o sistema de rotação batata/ grãos/forrageira e verificaram que as propriedades biológicas do solo (massa microbiana e quociente microbiano), foram influenciadas negativamente pela cultura da batata. Mas, a rotação com grãos e forrageira, possibilitou o aumento do teor de matéria orgânica, recuperando os atributos biológicos do solo. A rotação de culturas com adubos verdes pode ser utilizada como uma parte do programa manejo de solos com a intenção de reverter a degradação do solo (Grandy et al., 2002).

No entanto, além do manejo nutricional, o cultivo orgânico da batata depende da sanidade da cultura em relação às pragas e doenças para obtenção de melhores produtividades (Darolt et al., 2008). Para tanto, há duas alternativas básicas: o controle com produtos alternativos ou a utilização de cultivares resistentes. Algumas pesquisas apontam resultados satisfatórios no controle de pragas e doenças com produtos alternativos: Rauber et al. (2007) avaliaram a incidência de doenças Phythphora infestans (requeima) e Alternaria solani (pinta-preta), e insetos Diabrotica speciosa, Epitrix sp. com aplicação de preparados homeopáticos. Medeiros et al. (2008), pesquisaram o controle alternativa da requeima (Phytophthora infestans) em batata cultivada em sistema de base agroecológica e verificaram que a calda bordalesa $1 \%$ e o extrato de própolis $0,3 \%$ foram eficientes no controle da doença. Gomes et al. (2009), verificaram que a aplicação de silício contribuiu para a redução de injúrias de dois importantes desfolhadores, Diabrotica speciosa e Liriomyza spp., sem influenciar a produtividade da batateira em sistema orgânico.

Em relação a cultivares adaptadas ao sistema de cultivo orgânico, os produtores rurais dispõem de poucas informações a esse respeito. Desse modo, o objetivo neste trabalho foi avaliar, sob sistema de cultivo orgânico e em condições de campo, o potencial produtivo e a qualidade de genótipos nacionais e estrangeiros de batata, desenvolvidos para o cultivo convencional.

\section{MATERIAL E MÉTODOS}

O experimento foi conduzido em área agroecológica no Pólo Regional de Desenvolvimento Tecnológico dos Agronegócios do Leste Paulista, da Agência Paulista de Tecnologia dos Agronegócios (APTA), da Secretaria de Agricultura e Abastecimento do estado de São Paulo, com sede em Monte Alegre do Sul-SP (22 $41^{\circ}$ S, 46 $46^{\circ}$ W e $777 \mathrm{~m}$ de altitude).

O delineamento experimental utilizado foi em blocos ao acaso, com 18 tratamentos e quatro repetições. Cada parcela foi constituída por quatro linhas de $5 \mathrm{~m}$ de comprimento espaçadas de 80 $\mathrm{cm}$, com $25 \mathrm{~cm}$ entre batatas-semente, ou seja, cada parcela foi composta por 80 batatas-semente. Os tratamentos constaram dos seguintes genótipos: Agata, Asterix, Caesar, Cupido, Éden, Melody, Novella e Vivaldi, de origem estrangeira; e Apuã (IAC-5977), Aracy (IAC-2), Catucha (Epagri-361), IAC Aracy Ruiva, Itararé (IAC-5986), Monte Alegre 172, IAC 6090 (Ibituaçú), APTA 16.5, APTA 15.20 e APTA 21.54, todos nacionais.

$\mathrm{Na}$ caracterização química do solo da área foram observados os seguintes resultados: $\mathrm{MO}=20 \mathrm{~g} \mathrm{dm}^{-3} ; \mathrm{pH}\left(\mathrm{CaCl}_{2}\right)=$ 5,$1 ; \mathrm{P}=1 \mathrm{mg} \mathrm{dm}^{-3} ; \mathrm{K}=1,5 \mathrm{mmol}_{\mathrm{c}} \mathrm{dm}^{-3}$; $\mathrm{Ca}=21 \mathrm{mmol}_{\mathrm{c}} \mathrm{dm}^{-3} ; \mathrm{Mg}=11 \mathrm{mmol}_{\mathrm{c}} \mathrm{dm}^{-3}$; $\mathrm{H}+\mathrm{Al}=31 \mathrm{mmol}_{\mathrm{c}} \mathrm{dm}^{-3} ; \mathrm{CTC}=64,5$ $\mathrm{mmol}_{\mathrm{c}} \mathrm{dm}^{-3} ; \mathrm{V}=52 \% ; \mathrm{S}=8 \mathrm{mg} \mathrm{dm}^{-3} ; \mathrm{B}=$ $0,21 \mathrm{mg} \mathrm{dm}^{-3} ; \mathrm{Cu}=1,6 \mathrm{mg} \mathrm{dm}^{-3} ; \mathrm{Fe}=42$ $\mathrm{mg} \mathrm{dm}{ }^{-3} ; \mathrm{Mn}=15,0 \mathrm{mg} \mathrm{dm}^{-3} ; \mathrm{Zn}=3,0 \mathrm{mg}$ $\mathrm{dm}^{-3}$. A área foi cultivada inicialmente com adubação verde, semeada em 27 de dezembro de 2007. Foi utilizada a mistura de Sorgo forrageiro cultivar
Santa Elisa (20 sementes por metro linear) e Crotalaria juncea (25 sementes por metro linear), na proporção de 2:1 e espaçamento de $50 \mathrm{~cm}$ entre linhas. $\mathrm{O}$ adubo verde foi triturada em 16 de abril de 2008, incorporando-se ao solo $20 \mathrm{t}$ $\mathrm{ha}^{-1}$ de massa seca vegetal da mistura, que apresentou os seguintes teores de macronutrientes: $\mathrm{N}=10,3 \mathrm{~g} \mathrm{~kg}^{-1}, \mathrm{P}=1,2$ $\mathrm{g} \mathrm{kg}^{-1}, \mathrm{~K}=16,7 \mathrm{~g} \mathrm{~kg}^{-1}, \mathrm{Ca}=3,6 \mathrm{~g} \mathrm{~kg}^{-1}$, $\left.\mathrm{Mg}=4,4 \mathrm{~g} \mathrm{~kg}^{-1}\right)$. A fosfatagem e adubação foram efetuadas no sulco de plantio e por metro linear constou de 60 gramas de termofosfato, que adicionou ao solo $120 \mathrm{~kg} \mathrm{ha}^{-1} \mathrm{P}_{2} \mathrm{O}_{5} ; 135 \mathrm{~kg} \mathrm{ha}^{-1} \mathrm{Ca} ; 52,50$ $\mathrm{kg} \mathrm{ha}^{-1} \mathrm{Mg} ; 0,75 \mathrm{~kg} \mathrm{ha}^{-1} \mathrm{~B} ; 0,38 \mathrm{~kg} \mathrm{ha}^{-1}$ $\mathrm{Cu} ; 1,13 \mathrm{~kg} \mathrm{ha}^{-1} \mathrm{Mn} ; 75 \mathrm{~kg} \mathrm{ha}^{-1} \mathrm{Si} ; 4,13$ $\mathrm{kg} \mathrm{ha}^{-1} \mathrm{Zn}$ e $2 \mathrm{~kg}$ de composto orgânico, com teores de: $50 \mathrm{~g} \mathrm{~kg}^{-1} \mathrm{MO}$; $1,5 \mathrm{~g} \mathrm{~kg}^{-1}$; $\mathrm{N} ; 0,80 \mathrm{~g} \mathrm{~kg}^{-1} \mathrm{P}_{2} \mathrm{O}_{5}$ (ácido cítrico); $1,2 \mathrm{~g}$ $\mathrm{kg}^{-1} \mathrm{~K}_{2} \mathrm{O} ; 3,1 \mathrm{~g} \mathrm{~kg}^{-1} \mathrm{Ca}$; 0,6 g kg-1 $\mathrm{Mg}$; $0,6 \mathrm{~g} \mathrm{~kg}^{-1} \mathrm{~S}$.

As batatas-semente classe III, ou seja, com 30 a $40 \mathrm{~mm}$ de diâmetro (Brasil, 1987), foram semeadas em 17 de junho de 2008. Durante o cultivo foram feitos a amontoa e irrigações por aspersão como preconizado para a cultura. Não foi efetuada adubação de cobertura.

Em relação ao ataque de inseto-praga, foi detectada durante o monitoramento a presença de Epicauta atomaria, mas o número de indivíduos era muito baixo, o que não justificou o seu controle. Não foram adotadas medidas para manejo fitossanitário visando o controle de doenças fúngicas foliares, sendo que as plantas apresentaram sintomas de pinta-preta (Alternaria solani). $\mathrm{Na}$ leitura de infecção pelo fungo, realizada aos 70 dias após a emergência da batata, utilizou-se método desenvolvido por Reifschneider et al. (1983), no qual atribui-se notas de acordo com a porcentagem de área foliar infectada da parcela, adaptando-se as classes para: $1<2,5 \%, 2=2,5$ a $25 \%, 3=26$ a $50 \%$, $4=51$ a $75 \%$ e $5=>76 \%$.

A colheita foi iniciada em 30 de outubro e finalizada em sete de novembro de 2008, respeitando-se o ciclo de cada genótipo, sendo todas as parcelas do mesmo genótipo colhidas no mesmo dia, ou seja, 10 dias após a seca da rama. Foram avaliados a produtividade total (PT); produtividade comercial (PC); número de tubérculos total (NTT); 
número de tubérculos comercializáveis (NTC) ou seja, aqueles que apresentaram diâmetro transversal superior a $40 \mathrm{~mm}$; massa média dos tubérculos da produtividade total (MTT) e massa média dos tubérculos comercializáveis (MTC); e teor de matéria seca (MS) nos tubérculos. A matéria seca foi obtida em seis tubérculos da classe $>60 \mathrm{~mm}$ de diâmetro transversal, por parcela, por meio da secagem dos tubérculos em estufa com circulação forçada de ar, a $65^{\circ} \mathrm{C}$, até a obtenção de massa constante, sendo o teor, em porcentagem, calculado pela fórmula: (massa seca/massa fresca) x 100 .

Para atendimento das pressuposições da análise de variância, os dados de produtividade total foram transformados em $\log (\mathrm{X})$. A escala logarítmica foi a que melhor adequou os dados para que os mesmos apresentassem normalidade. As médias foram agrupadas pelo teste de Scott-Knott em nível de 5\% de probabilidade (Scott \& Knott, 1974). Para análise da severidade da pinta-preta foi utilizado o teste não paramétrico de Friedman em nível de $5 \%$ de probabilidade.

\section{RESULTADOS E DISCUSSÃO}

Os 18 genótipos formaram quatro grupos distintos para a produtividade total (PT), comercial (PC), massa média dos tubérculos da produtividade total (MTT) e massa média dos tubérculos da produtividade comercial (MTC) (Tabela 1). Quanto ao PT os quatro grupos foram constituídos de 7, 6, 3 e 2 genótipos, respectivamente, mostrando que $50 \%$ dos genótipos apresentaram produtividade acima de $14 \mathrm{t} \mathrm{ha}^{-1}$. As maiores PT foram apresentadas pelos genótipos APTA 16.5, APTA 21.54, IAC 6090 (Ibituaçú), Cupido, Apuã, Itararé e Monte Alegre 172. As menores PT foram apresentadas pelas cultivares Vivaldi e Éden.

O clone APTA 21.54, representando o primeiro grupo, com PC de 18,07 tha-1, superou os demais grupos formados por 4, 5 e 8 genótipos. Os genótipos APTA 16.5, Cupido, IAC 6090 (Ibituaçú) e Itararé, formaram o segundo grupo em relação a PC. Souza et al. (2007) avaliaram o rendimento de três cultivares de batata (Itararé, Monte Bonito e Ma- tilda) em condições climáticas que não permitiram a ocorrência de requeima durante todo o ciclo, apresentando a cultivar Itararé a maior produtividade comercial, com 17,53 tha-1. Este resultado e o do presente estudo comprovam o potencial da cultivar Itararé em sistemas orgânicos.

Ramos (2008) relatou que em Itararé-SP, no Pólo APTA do Sudoeste Paulista, na safra das secas de 1999, em sistema convencional, os genótipos Ibituaçú, Itararé, Cupido, Aracy e Aracy Ruiva apresentaram produtividade de 38,$3 ; 31,6 ; 26,6,25,0$ e $24,6 \mathrm{t} \mathrm{ha}^{-1}$, respectivamente. Feltran (2005) obteve 33,20 $\mathrm{t} \mathrm{ha}^{-1}$ com a cultivar Agata em sistema convencional, enquanto Melo et al. (2003) relatam produtividade comercial de $60 \mathrm{t} \mathrm{ha}^{-1}$ para 'Agata' no estado de São Paulo. Desse modo, as produtividades da batata em sistemas convencionais têm sido superiores à dos sistemas orgânicos, devido, ao que tudo indica, ao maior "input" de insumos, principalmente fertilizantes, inseticidas e fungicidas empregados nos sistemas de cultivo convencionais. Maggio et al. (2008) reportaram a redução de $25 \%$ da produtividade do sistema orgânico de produção em relação ao convencional.

Darolt et al. (2008) relataram que para se obter altas produtividades na cultura da batata em sistema convencional o custo total de insumos foi $81 \%$ superior em relação ao sistema orgânico. Ao analisarem comparativamente os sistemas orgânico e convencional de produção de batata, verificaram que os preços pagos ao produtor orgânico foram, em média, 90\% superiores aos pagos pelos tubérculos produzidos convencionalmente. Portanto, os custos de produção mais baixos, aliados aos preços mais

Tabela 1. Produtividade total de tubérculos (PT), produtividade comercial de tubérculos (PC), massa média dos tubérculos da produtividade total (MTT) e massa média dos tubérculos comercializáveis (MTC) e porcentagem da matéria seca dos tubérculos (MS) dos 18 genótipos de batata avaliados em sistema orgânico de produção (total tuber yield (PT), marketable tuber yield (PC), average mass of tubers (MTT) and average mass of marketable tubers (MTC) and percentage of dry matter of tubers (MS) in potato genotypes). Monte Alegre do Sul, APTA, 2008.

\begin{tabular}{|c|c|c|c|c|c|c|c|}
\hline \multirow{3}{*}{$\begin{array}{l}\text { Genótipo } \\
\text { APTA } 16.5\end{array}$} & \multicolumn{2}{|l|}{ PT } & PC & MTT & \multicolumn{2}{|c|}{ MTC } & \multirow{2}{*}{$\begin{array}{l}\text { MS } \\
(\%)\end{array}$} \\
\hline & \multicolumn{3}{|c|}{$\left(\mathrm{t} \mathrm{ha}^{-1}\right)$} & \multicolumn{3}{|c|}{ (g/tubérculo) } & \\
\hline & 24,25 & $\mathrm{a}$ & $15,59 \mathrm{~b}$ & $33,68 \mathrm{c}$ & 63,02 & $\mathrm{c}$ & 22,86 \\
\hline APTA 21.54 & 23,21 & $\mathrm{a}$ & $18,07 \mathrm{a}$ & $38,61 \mathrm{~b}$ & 67,17 & $\mathrm{~b}$ & 23,11 \\
\hline IAC 6090 (Ibituaçú) & 20,19 & $\mathrm{a}$ & $13,69 \mathrm{~b}$ & $32,80 \mathrm{~d}$ & 62,10 & $\mathrm{c}$ & 28,26 \\
\hline Cupido & 18,03 & $\mathrm{a}$ & $14,52 \mathrm{~b}$ & 50,17 a & 75,21 & $\mathrm{a}$ & 21,85 \\
\hline Apuã & 17,08 & $\mathrm{a}$ & $7,86 \mathrm{c}$ & $26,39 \mathrm{~d}$ & 50,80 & $d$ & 22,19 \\
\hline Itararé & 16,66 & $\mathrm{a}$ & $13,52 \mathrm{~b}$ & 49,65 a & 78,08 & $\mathrm{a}$ & 23,60 \\
\hline Monte Alegre 172 & 16,18 & a & $8,15 \mathrm{c}$ & $26,32 \mathrm{~d}$ & 52,65 & d & 23,61 \\
\hline Caesar & 15,31 & $\mathrm{~b}$ & $10,24 \mathrm{c}$ & $32,95 \mathrm{c}$ & 66,35 & $\mathrm{~b}$ & 20,50 \\
\hline Aracy Ruiva & 14,64 & $\mathrm{~b}$ & $9,22 \mathrm{c}$ & $33,98 \mathrm{c}$ & 61,46 & $\mathrm{c}$ & 26,25 \\
\hline Melody & 13,48 & $\mathrm{~b}$ & $5,17 \mathrm{~d}$ & $23,97 \mathrm{~d}$ & 53,57 & $\mathrm{~d}$ & 21,05 \\
\hline Asterix & 12,28 & $\mathrm{~b}$ & $9,02 \mathrm{c}$ & $38,25 \mathrm{~b}$ & 64,17 & $\mathrm{c}$ & 22,13 \\
\hline APTA 15.20 & 12,11 & $\mathrm{~b}$ & $5,64 \mathrm{~d}$ & $21,81 \quad \mathrm{c}$ & 54,76 & $\mathrm{~d}$ & 24,73 \\
\hline Aracy & 11,31 & $\mathrm{~b}$ & $6,38 \mathrm{~d}$ & $27,39 \mathrm{~d}$ & 57,85 & $\mathrm{~d}$ & 26,40 \\
\hline Catucha & 9,68 & $\mathrm{c}$ & $6,20 \mathrm{~d}$ & $33,52 \mathrm{c}$ & 62,51 & $\mathrm{c}$ & 24,90 \\
\hline Novella & 8,66 & $\mathrm{c}$ & $5,44 \mathrm{~d}$ & $23,95 \mathrm{c}$ & 57,83 & $\mathrm{~d}$ & 20,33 \\
\hline Agata & 8,37 & $\mathrm{c}$ & $4,55 \mathrm{~d}$ & $24,94 \mathrm{~d}$ & 57,03 & $\mathrm{~d}$ & 19,16 \\
\hline Vivaldi & 6,27 & $\mathrm{~d}$ & $3,40 \mathrm{~d}$ & $26,73 \mathrm{~d}$ & 60,13 & $\mathrm{c}$ & 19,12 \\
\hline Éden & 6,14 & $\mathrm{~d}$ & $3,78 \mathrm{~d}$ & $28,55 \mathrm{~d}$ & 60,71 & $\mathrm{c}$ & 22,29 \\
\hline $\mathrm{CV}(\%)$ & 2,77 & & 23,11 & 12,14 & 7,57 & & \\
\hline
\end{tabular}

Médias seguidas de mesma letra na coluna pertencem ao mesmo agrupamento pelo Teste de Scott-Knott, $\mathrm{p}>0,05$ (means followed by the same letter in column are not different, Scott-Knott test, $\mathrm{p}>0,05$ ). 
Tabela 2. Severidade da pinta-preta (Alternaria solani) aos 60 dias após a emergência (DAE) em 18 genótipos de batata avaliados em sistema orgânico de produção (severity of potato early blight (Alternaria solani) at 60 days after emergency (DAE) in 18 potato genotypes under organic cultivation). Monte Alegre do Sul, APTA, 2008.

\begin{tabular}{|c|c|c|c|}
\hline \multirow{2}{*}{ Genótipo } & \multirow{2}{*}{$\begin{array}{c}\text { Mediana } \\
\text { Escala de notas: } 1 \text { a } 5^{*}\end{array}$} & \multirow{2}{*}{$\begin{array}{c}\text { Valor } \\
\text { mínimo }\end{array}$} & \multirow{2}{*}{$\begin{array}{c}\text { Valor } \\
\text { máximo }\end{array}$} \\
\hline & & & \\
\hline APTA 16.5 & $1,0 \quad b$ & 1 & 1 \\
\hline Apuã & $1,0 \quad \mathrm{~b}$ & 1 & 5 \\
\hline Aracy & $1,0 \quad \mathrm{~b}$ & 1 & 1 \\
\hline Aracy Ruiva & $1,0 \quad b$ & 1 & 1 \\
\hline Éden & $1,0 \quad b$ & 1 & 1 \\
\hline IAC 6090 (Ibituaçú) & $1,0 \quad b$ & 1 & 1 \\
\hline Monte Alegre 172 & $1,0 \mathrm{~b}$ & 1 & 1 \\
\hline APTA 21.54 & $3,0 \mathrm{ab}$ & 3 & 3 \\
\hline Catucha & $3,0 \mathrm{ab}$ & 3 & 3 \\
\hline Cupido & $3,0 \mathrm{ab}$ & 3 & 5 \\
\hline Itararé & $3,0 \mathrm{ab}$ & 3 & 4 \\
\hline Agata & $3,5 \mathrm{ab}$ & 3 & 4 \\
\hline Novella & $3,5 \mathrm{ab}$ & 3 & 4 \\
\hline APTA 15.20 & $4,0 \mathrm{ab}$ & 3 & 5 \\
\hline Caesar & $4,0 \mathrm{ab}$ & 4 & 4 \\
\hline Melody & $4,0 \mathrm{ab}$ & 3 & 5 \\
\hline Vivaldi & $4,0 \mathrm{ab}$ & 3 & 4 \\
\hline Asterix & $5,0 \quad \mathrm{a}$ & 3 & 5 \\
\hline
\end{tabular}

Medianas seguidas de mesma letra não diferem entre si pelo teste de Friedman, $p>0,05$ (medians followed by the same letter are not different, Friedman test, $\mathrm{p}>0,05$ ); *Escala de notas: $1<2,5 \%, 2=2,5$ a $25 \%, 3=26$ a $50 \%, 4=51$ a $75 \%$ e $5 \geq 76 \%$ (scale of grades: $1<2,5 \%$, $2=2,5$ a $25 \%, 3=26$ a $50 \%, 4=51$ a $75 \%$ e $5 \geq 76 \%$ ).

elevados na hora da comercialização, podem compensar a menor produção obtida pelo sistema orgânico.

Em relação à massa média dos tubérculos da produtividade total (MTT) os grupos foram constituídos de 2, 2, 6 e 8 genótipos. As maiores MTT foram apresentadas pelas cultivares Itararé e Cupido, sendo o segundo grupo formado por 'APTA 21.54' e 'Asterix'. Os grupos diferenciados pelo teste de Scott-Knott para MTC foram constituídos de 2, 2, 7 e 7 genótipos, sendo que 'Cupido' e 'Itararé' formam o primeiro grupo, com maiores MTC, e 'APTA 21.54' e 'Caesar' formam o segundo grupo. Convém destacar que, atualmente, devido à baixa oferta de batata orgânica, praticamente toda produção tem sido comercializada, independente do tamanho do tubérculo.

Pelo presente estudo, as cultivares holandesas Asterix, Caesar, Melody, Novella e Vivaldi, e a cultivar francesa produzidos em sistema convencional no município de São Manuel, na safra das águas do ano 2000, e obtiveram os seguintes teores em relação à matéria seca: Agata (16,4\%), Apuã (18,4\%), Asterix (19,1\%), Aracy (20,3\%), Itararé (20,5\%), IAC Aracy Ruiva (20,6\%) e Ibituaçú $(22,4 \%)$. Os teores de matéria seca encontrados no presente estudo são mais elevados do que os reportados por Feltran (2004). É possível que esse fato esteja relacionado à interação entre genótipo e ambiente, uma vez que são comuns tais variações quando uma determinada cultivar é submetida a diferentes sistemas e ambientes de cultivo (Cacace et al., 1994; Melo, 1999).

Em relação à severidade da pinta-preta, as cultivares Apuã, Aracy, Aracy Ruiva, Éden, Monte Alegre 172 e os clones Ibituaçú e APTA 16.5 apresentaram poucos ou ausência de sintomas (Tabela 2). A cultivar Asterix foi severamente infectada pela pinta-preta. As demais cultivares apresentaram resistência intermediária, variando de 26 a 50 a porcentagem de área foliar infectada para as cultivares Catucha, Cupido, Itararé, Agata, Novella e clone APTA 21.54. Já as cultivares Melody, Vivaldi, Caesar e o clone APTA 15.20 apresentaram de 51 a $75 \%$ da área foliar afetada pela doença (Tabela 2). A pinta-preta pode tornar-se muito destrutiva quando encontra condições de alta temperatura $\left(25\right.$ a $\left.30^{\circ} \mathrm{C}\right)$ e umidade relativa do ar próxima a $90 \%$ (Maffia et al., 1980). Sabe-se que, em geral, as cultivares de maturidade precoce mostram-se mais suscetíveis à doença que as tardias (Brune et al., 1994), o que pode ser observado nas cultivares holandesas: Asterix, Vivaldi, Melody, Caesar, Novella, Agata e Cupido (Tabela 2).

As cultivares Itararé, Apuã e Cupido têm potencial para serem recomendadas para sistemas de cultivo orgânico. Os clones avançados, APTA 16.5, APTA 21.54 e IAC 60-90 (Ibituaçú), obtidos pelo setor público, reúnem características vantajosas para o segmento da bataticultura orgânica.

\section{AGRADECIMENTOS}

superior a $24 \%$ produzem fatias quebradiças e causam desgaste excessivo dos fatiadores (Melo \& Yorinori, 2000). Feltran et al. (2004), avaliaram a qualidade tecnológica de genótipos de batatas
À Agência Paulista de Tecnologia dos Agronegócios (APTA), Pólos Regionais Leste Paulista (Monte Alegre do 
Sul-SP) e Centro-Sul (Piracicaba-SP), à estagiária Laís Ferraz de Camargo, e ao CNPq pela concessão da bolsa de doutorado.

\section{REFERÊNCIAS}

BRASIL. Portaria n.154, de 24 de julho de 1987. Dispõe sobre as normas gerais para certificação de batata-semente. Diário Oficial, Brasília, 24 jul. 1987. Seção 1, p.11804.

BRUNE S; MELO PE; LIMA MF. 1994. Resistência a Alternaria solani, características agronômicas e qualidade de fritura em clones de batata imunes a PVX e PVY. Horticultura Brasileira 12: 125-130.

CACACE JE; HUARTE MA; MONTI MC. 1994. Evaluation of potato cooking quality in Argentina. American Potato Journal 71: 145-153.

DAROLT MR; RODRIGUES A; NAZARENO N; BRISOLLA A; RÜPPEL O. 2008. Análise comparativa entre o sistema orgânico e convencional de batata comum. Disponível em: <http://www.planetaorganico.com/ Daroltbatata.htm>. Acesso em: 4 dez. 2008.

FAO. 2008. Potatoes, nutrition and diet. Disponível em: <http://www.potato2008.org/ en/potato/IYP-6en.pdf/perspectives/index. $\mathrm{html}>$. Acesso em: 17 jan. 2011.

FELTRAN JC. 2005. Adubação mineral na cultura da batata e do residual no feijoeiro. Botucatu: UNESP. 112p (Tese doutorado).

FELTRAN JC; LEMOS LB; VIEITES RL. 2004. Technological quality and utilization of potato tubers. Sciencia Agricola 61: 593-597.

GOMES FB; MORAES JC; NERI DKP. 2008. Adubação com silício como fator de resistência a insetos-praga e promotor de produtividade em cultura de batata inglesa em sistema orgânico. Ciência agrotécnica 33: 18-23.

GRANDY AS; PORTER GA; ERICH MS. 2002. Organic amendment and rotation crop effects on the recovery of soil organic matter and aggregation in potato cropping systems. Soil Science Society of America Journal 66: 1311-1319.

IBGE. Produção agrícola municipal 2009, lavoura temporária. Disponível em: <http://www.ibge. gov.br/estadosat>. Acesso em: 14 dez. 2010.

LOVE SL. 2000. Important quality characteristics in breeding processing potatoes. In: WORLD POTATO CONGRESS, 4., Wageningen Proceedings... Amsterdam: Wageningen Press, p.261-266.

MAFFIA LA; MARTINS MCP; MATSUOKA K. 1980. Doenças do tomateiro. Informe Agropecuário 6: 42-60.

MAGGIO A; CARILLO P; BULMETTI GS; FUGGI A; BARBIERI G; DE PASCALE S. 2008. Potato yield and metabolic profiling under conventional and organic farming. Europe Journal Agronomy 28: 343-350.

MEDEIROS CAB; STRASSBURGER AS; GOMES CB; WOLFF LF. 2008. Controle alternativo de requeima (Phytophthora infestans) em batata cultivada em sistema de base ecológica. Horticultura Brasileira 26: S4821-S4825.

MELO PCT; GRANJA NP; MIRANDA FILHO HS; SUGAWARA AC; OLIVEIRA RF. 2003. Análise do crescimento da cultivar de batata Agata. Horticultura Brasileira, Suplemento 21: 323-324.

MELO PE. 1999. Cultivares de batata potencialmente úteis para processamento na forma de fritura no Brasil e manejo para obtenção de tubérculos adequados. Informe Agropecuário 20: 112-119.

MELO PE; YORINORI NA. 2000. Apresentação das instituições e temas técnicos: comentários e apartes. In: MELO PE; BRUNE S. (ed). In:
WORKSHOP BRASILEIRO DE PESQUISA EM MELHORAMENTO DE BATATA, 1., 1996, Londrina. Anais... Brasília: Embrapa Hortaliças 3.1: 81-108.

NELSON, KL; LYNCH DH; BOITEAU G. Assessment of changes in soil health throughout organic potato rotation sequences. 2009. Agriculture, Ecosystems and Environment 131: 220-228.

RAMOS VJ. 2008. Características dos cultivares de batata IAC-APTA. In: ENCONTRO REGIONAL DA BATATA: PRODUÇÃO E SUSTENTABILIDADE, 5., Itararé. Palestra.. Itararé: Pólo APTA do Sudoeste Paulista.

RAUBER LP; BOFF MI; SILVA Z; FERREIRA A; BOFF P. 2007. Manejo de doenças e pragas da batateira pelo uso de preparados homeopáticos e variabilidade genética. Revista Brasileira de Agroecologia 2: 1008-1011.

REIFSCHNEIDER FJB; SIQUEIRA CB; CORDEIRO CMT. 1983. Índice de doenças de hortaliças no Brasil - Bactérias e Fungos. Brasília: EMBRAPA. 156p.

SCOTT AJ; KNOTT M. 1974. A cluster analysis method for grouping means in the analysis of variance. Biometrics 30: 507-512.

SILVA TO; MENEZES RSC; TIESSEN H; SAMPAIO EVSB; SALCEDO IH; SILVEIRA LM. 2007. Adubação orgânica da batata com esterco e, ou, Crotalaria juncea: I produtividade vegetal e estoque de nutrientes no solo em longo prazo. Revista Brasileira de Ciência do Solo 31: 39-49.

SOUZA JL; VENTURA JA; COSTA H. 2007. Avaliação de genótipos de batata (Solanum tuberosum) em cultivo orgânico. Disponível em: <http://www.cnph.embrapa.br/novidade/ eventos/organica/Anexre05.doc $>$. Acesso em: 13 ago. 2007.

VAN DELDEN A. 2001. Yield and growth components of potato and wheat under organic nitrogen management. Agronomy Journal 93: 1370-1385. 